\title{
Analyzing and strengthening the vaccine safety program in Manitoba
}

\author{
Montalban $\mathrm{JM}^{1^{\star}}$, Ogbuneke $\mathrm{C}^{2}$, Hilderman $\mathrm{T}^{1,3}$ \\ ${ }^{1}$ Public Health Branch, Manitoba Health, Healthy Living and Seniors, Winnipeg, MB \\ ${ }^{2}$ Public Health and Preventive Medicine, Northern Ontario School of Medicine, Sudbury, ON \\ ${ }^{3}$ Community Health Sciences, University of Manitoba Faculty of Medicine, Winnipeg, MB
}

"Corresponding author: joselito.montalban@gmail.com

\section{Abstract}

Background: The emergence of a novel influenza A virus in 2009 and the rapid introduction of new pandemic vaccines prompted an analysis of the current state of the adverse events following immunization (AEFI) surveillance response in several provinces.

Objectives: To highlight aspects of the situational analysis of the Manitoba Health, Healthy Living and Seniors (MHHLS's) AEFI surveillance system and to demonstrate how common business techniques could be usefully applied to a provincial vaccine safety monitoring program.

Method: Situational analysis of the AEFI surveillance system in Manitoba was developed through a strengthsweaknesses-opportunities-threats (SWOT) analysis and informed by the National Immunization Strategy vaccine safety priorities. Strategy formulation was developed by applying the threats-opportunities-weaknesses-strengths (TOWS) matrix.

Results: Thirteen strategies were formulated that use strengths to either take advantage of opportunities or avoid threats, that exploit opportunities to overcome weaknesses, or that rectify weaknesses to circumvent threats. These strategies entailed the development of various tools and resources, most of which are either actively underway or completed.

Conclusion: The SWOT analysis and the TOWS matrix enabled MHHLS to enhance the capacity of its vaccine safety program.

\section{Introduction}

In late April of 2009, the World Health Organization (WHO) announced the emergence of a novel influenza A virus (1). In response, new vaccines against the pandemic strain had to be rapidly introduced with limited safety information in humans. At that time, Canada activated its Pandemic Influenza Plan for the Health Sector (2). Although this document addressed issues concerning vaccines, vaccine safety was only sparingly dealt with.

At the end of the pandemic, the Vaccine Vigilance Task Group- an expanded version of the Vaccine Vigilance Working Group (3) that included more medical and epidemiological expertise- commissioned an evaluation of the federal, provincial and territorial adverse event following immunization (AEFI) surveillance response (4). In line with this work, commencing in October 2010, Manitoba Health, Healthy Living and Seniors (MHHLS) signed a memorandum of agreement with the Public Health Agency of Canada (PHAC) to embark on a vaccine safety best practice model (BPM) pilot project. The BPM pilot was intended to bridge the gaps between current practice and the vaccine safety priorities or specific objectives enunciated in the National Immunization Strategy (5) to optimize 
the vaccine safety system, to maintain professional and public confidence in the safety of vaccines, and to address growing anti-immunization concerns.

Two common business tools were used to determine the way to bridge those gaps: the SWOT (strengthsweaknesses-opportunities-threats) analysis, and the TOWS (threats-opportunities-weaknesses-strengths) matrix.

The SWOT analysis is a simple way of conducting an audit of the factors that affect an organization. It facilitates the assessment of the internal strengths and weaknesses of an organization-in this case, MHHLS. Likewise, the SWOT analysis facilitates examination of the opportunities and threats, which are factors in the external environment. It is imperative to make a distinction between internal and external factors as the organization naturally has more control over the former than the latter. Any appraisal of an organization's current situation should take into account the issues that are extrinsic as well as intrinsic to it.

The TOWS matrix is a useful tool for generating strategies or action steps in health management (6) and continues where the SWOT analysis leaves off (7). It allows for systematically matching or aligning the different elements itemized in the SWOT analysis, showing the relationships among the internal and external factors, thereby helping planners formulate measures that use strengths to either take advantage of opportunities or avoid threats, that exploit opportunities to overcome weaknesses, or that rectify weaknesses to circumvent threats.

A literature review of MEDLINE using the search terms "SWOT," "TOWS," "immunization," and "vaccine" revealed that, although these techniques have been used worldwide in strategic planning for immunization programs, only a handful of scholarly articles have actually been published that give an account of their application in this area. For example, one or both tools were utilized to address issues with vaccine shortage policies in the United States (7) and to guide a smallpox revaccination program in Israel (8).

The objectives of this study were to highlight aspects of the situational analysis of MHHLS's AEFI surveillance system and to demonstrate how common business techniques could be usefully applied to a provincial vaccine safety monitoring program.

\section{Method}

As part of the situational analysis of the current state of MHHLS's AEFI surveillance program at the early stages of this pilot project, the SWOT analysis was applied to map out the different factors affecting the organization. These factors were identified based on responses to questionnaires mailed out to various health professionals involved in AEFI surveillance, including managers of hospital emergency rooms, managers of long-term care facilities, epidemiologists, regional medical officers of health (MOHs) and other physicians, and immunization coordinators. Subsequently, the TOWS matrix was applied to generate or prioritize strategies identified in consultation with a vaccine safety advisory group composed of provincial AEFI surveillance personnel and infectious disease experts.

\section{Results}

A list of strengths, weaknesses, opportunities and threats was developed (see Table 1). Application of the TOWS matrix to this list enabled identification of strategies, which necessitated the development of various tools and resources. 
Table 1: List of strengths, weaknesses, opportunities and threats (SWOT) for the MHHLS ${ }^{1}$ best practice model for vaccine safety

\begin{tabular}{|c|c|}
\hline INTERNAL & EXTERNAL \\
\hline $\begin{array}{l}\text { Strengths } \\
\text { - Existing reporting form } \\
\text { - } \text { Availability of administrative databases } \\
\text { - } \text { Existing risk assessment procedure for public } \\
\text { - Ubalth alerts } \\
\text { - } \quad \text { Provinitous provincial government website }\end{array}$ & $\begin{array}{cl}\text { Threats } & \\
\text { - } & \text { Erratic reporting } \\
\text { - } & \text { Illegible and incorrectly filled out forms } \\
& \text { authorities as to what reviews occur at the } \\
& \text { provincial level } \\
\text { - } & \text { Poor communication among stakeholders }\end{array}$ \\
\hline 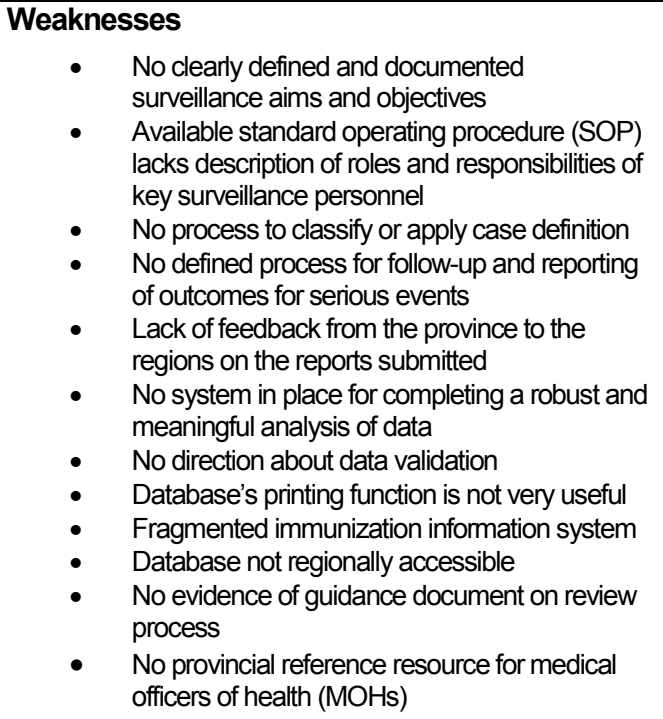 & 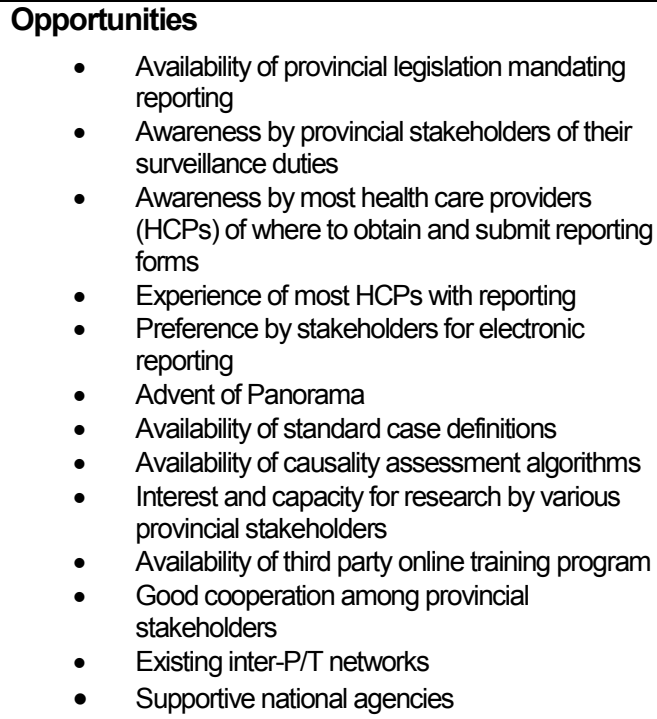 \\
\hline
\end{tabular}

\section{Resources identified from the strengths-opportunities strategy}

Online education program for AEFI. By leveraging the credibility afforded to it by its mandate as the official policymaking body for the province's public health system, MHHLS is able to arrange with a provider of online continuing professional education free access for health care providers (HCPs) to an existing online immunization training course (9). This course includes a module on AEFI.

\section{Resources identified from the weaknesses-opportunities strategies}

AEFI surveillance roles and responsibilities document. This relies on the fact that Manitoba has provincial legislation mandating HCPs to report AEFIs. This also counts on the cooperation and good will of provincial stakeholders.

Brighton assessment tool. This was developed by PHAC, and adapted by MHHLS, based on AEFI case definitions established by the Brighton Collaboration (10).

Provincial AEFI causality assessment guide. This lays out a provincial process for follow-up of serious event outcomes by providing a standardized procedure specific to Manitoba for ascertaining the certainty of any potential causal association of vaccine administration with an AEFI that meets one or more of the seriousness criteria. It adheres to the Clinical Immunization Safety Assessment algorithm (11) and is currently being actively 
utilized for assessing individual AEFIs. It is concurrently being continuously developed, guided by the revised WHO AEFI causality assessment user manual (12), mainly to expand the algorithm to include how to deal with population-level AEFIs.

AEFI signal detection methodology. This will depend on the outcome of research being conducted by the University of Manitoba on behalf of MHHLS with respect to AEFI background rates and signal validation. Additionally, the Public Health Intelligence for Disease Outbreaks, a statistical tool developed by the British Columbia Centre for Disease Control (BCCDC), which MHHLS is already using for alerting outbreaks of certain reportable diseases, could be tapped.

Online AEFI tool linked directly to database. Given the preference by stakeholders for electronic AEFI reporting, tapping of the Pan-Canadian Public Health Communicable Disease Surveillance \& Management Project (Panorama) is being explored to rectify a fragmented immunization information system by having a single database for client immunization, vaccine inventory and AEFI surveillance records.

AEFI management aid. This would address the lack of a provincial reference resource to guide MOHs in making AEFI management recommendations and could emulate examples from other jurisdictions, such as the AEFI section of the BCCDC's Immunization Manual (13).

\section{Resources identified from the strengths-threats strategies}

Improved AEFI reporting form. A modified layout of the reporting form that is deemed more intuitive for health professionals was considered to prevent or minimize certain errors commonly made when filling out the form.

AEFI risk assessment tool. This will be used to assess risk and potential impact of an AEFI alert on the immunization program. It is based on an existing MHHLS risk assessment procedure for public health alerts that provides a structured approach to coordination of initial actions and communications following receipt of an alert indicating potential risk to human health.

AEFI page within the provincial government website. This web page (14) can serve as an avenue for disseminating AEFI-related information and resources in the face of what is perceived as prevailing poor communication among various provincial AEFI surveillance stakeholders.

\section{Resources identified from the weaknesses-threats strategies}

Periodic AEFI surveillance reports. These are seen as a way to mitigate a weakness with respect to feedback, or lack thereof, from the province to the regions on AEFI reports the latter submit, thereby addressing the perceived threat of health regions not being aware as to what reviews occur at the provincial level.

AEFI database printing function. This allows printing of individual database records in form view (as opposed to the line view printing function in Access, which is not very practicable) so that a fresh and clean copy of the AEFI report is generated directly from the provincial database and faxed to PHAC's Canadian AEFI Surveillance System (and others, such as MOHs, who require them), thereby circumventing the progressive degradation of paper reports with repeated printing and faxing as they move through the reporting process (reporter $\rightarrow \mathrm{MOH} \rightarrow$ $\mathrm{MHHLS} \rightarrow$ PHAC).

AEFI standard operating procedure (SOP) and user guide. These rectify the need for guidance documents on the reporting process, the user guide focusing mainly on AEFI reporters and the SOP on surveillance staff at MHHLS. These are expected to help prevent or minimize inconsistent reporting.

The resources developed from the TOWS matrix are summarized in Table 2. 
Table 2: Overview of the TOWS matrix for the MHHLS best practice model for vaccine safety

\begin{tabular}{|c|c|c|c|}
\hline & & \multicolumn{2}{|r|}{ INTERNAL } \\
\hline & & Strengths & Weaknesses \\
\hline \multirow{2}{*}{ EXTERNAL } & Opportunities & $\begin{array}{l}\text { - Online AEFI education } \\
\text { program }\end{array}$ & $\begin{array}{l}\text { - AEFI surveillance roles and responsibilities document } \\
\text { - } \text { Brighton assessment tool } \\
\text { - } \text { Provincial AEFI causality assessment guide } \\
\text { - } \text { AEFI signal detection methodology } \\
\text { - } \text { Online AEFI tool linked directly to database } \\
\text { AEFI management aid }\end{array}$ \\
\hline & Threats & $\begin{array}{l}\text { - } \text { Improved AEFI } \\
\text { reporting form } \\
\text { - } \text { AEFI risk assessment } \\
\text { tool } \\
\text { - } A E F I \text { web page }\end{array}$ & $\begin{array}{l}\text { - } \text { Periodic AEFI surveillance reports } \\
\text { - AEFI database printing function } \\
\text { - AEFI SOP and user guide }\end{array}$ \\
\hline
\end{tabular}

\section{Discussion and conclusion}

The TOWS matrix and SWOT analysis are tools developed originally for the business sector that, used together, can also provide a powerful approach to public health planning. MHHLS has benefitted from the insight gained from the application of these business techniques that paved the way for generating the tools and resources needed in building what is hoped will be a sound vaccine safety program that can meet prevailing as well as emerging challenges posed by the continuing expansion of its overall immunization program. Most of the foregoing strategies are either actively underway or completed and continuously being improved. The ones pertaining to tapping Panorama, developing a management aid and revising the reporting form are either on hold or yet to commence.

\section{Acknowledgements}

The Manitoba Health, Healthy Living and Seniors AEFI best practice model pilot project was funded by the Public Health Agency of Canada. Special thanks to Dr. Barbara Law, Chief of PHAC's Vaccine Safety Section, for her unfailing assistance and guidance.

\section{Conflict of interest}

None

\section{References}

(1) World Health Organization (WHO) Media Centre. Statement to the press by WHO Director-General Dr. Margaret Chan. World now at the start of 2009 influenza pandemic. Geneva: WHO; 2009 Jun 11 [2013 Apr 8]. http://www.who.int/mediacentre/news/statements/2009/h1n1_pandemic_phase6_20090611/en/index.html

(2) Public Health Agency of Canada (PHAC). The Canadian Pandemic Influenza Plan for the Health Sector. Ottawa: PHAC; 2006.

(3) Ahmadipour N, Toth E, Law BJ. Canada's Vaccine Vigilance Working Group. CCDR. CCDR 2014; 40 Suppl 3:S3740. 
(4) One World Inc., editor. Draft meeting proceedings: Vaccine Vigilance Task Group (VVTG); 2010 Mar 30-31. Ottawa: Public Health Agency of Canada [unpublished].

(5) Advisory Committee on Population Health and Health Security. National Immunization Strategy: Final Report 2003. A Report from the F/P/T Advisory Committee on Population Health and Health Security to the Conference of F/P/T Deputy Ministers of Health. Ottawa: Public Health Agency of Canada; 2004. http://www.phac-aspc.gc.ca/publicat/nis-sni-03/index-eng.php

(6) Proctor T. Strategic marketing management for health management: cross impact matrix and TOWS (threats, opportunities, weaknesses, strengths). Journal of Management in Medicine. 2000;14(1):47-56. Epub 2001/02/24. PubMed PMID: 11183998.

(7) Uscher-Pines L, Barnett DJ, Sapsin JW, Bishai DM, Balicer RD. A systematic analysis of influenza vaccine shortage policies. Public Health. 2008;122(2):183-91. Epub 2007/09/11. doi: 10.1016/j.puhe.2007.06.005. PubMed PMID: 17825858.

(8) Huerta M, Balicer RD, Leventhal A. SWOT analysis: Strengths, weaknesses, opportunities and threats of the Israeli smallpox revaccination program. The Israel Medical Association Journal. 2003;5(1):42-6. Epub 2003/02/21. PubMed PMID: 12592958.

(9) Canadian Paediatric Society. Immunization Competencies Education Program [cited 2014]. http://www.advancingpractice.com/p-68-immunization-competencies-education-program.aspx

(10) Brighton Collaboration. Case Definitions [cited 2014]. https://brightoncollaboration.org/public/resources/standards/case-definitions.html

(11) Halsey NA, Edwards KM, Dekker CL, Klein NP, Baxter R, LaRussa P, et al. Algorithm to assess causality after individual adverse events following immunizations. Vaccine. 2012;30:5791-8.

(12) Global Advisory Committee on Vaccine Safety. Causality assessment of an adverse event following immunization (AEFI): User manual for the revised WHO classification. Geneva: World Health Organization; 2013.

(13) British Columbia Centre for Disease Control (BCCDC). Communicable Disease Control Manual. Chapter 2. Immunization. Section IX-Adverse Events Following Immunization. Vancouver, BC: BCCDC; 2014 Jan [cited 2014 Jul 24]. http://www.bccdc.ca/NR/rdonlyres/D8098B26-CD7B-4A65-914CAA98B15CC004/0/SectionIX_AdverseEventsFollowingImmunizationsJanuary2014.pdf

(14) Manitoba Health, Healthy Living and Seniors. Communicable Disease Control. Adverse Events Following Immunization (AEFI). Winnipeg, MB: Province of Manitoba [cited 2014]. http://www.gov.mb.ca/health/publichealth/cdc/div/aefi.html 